\title{
GPCR and IR genes in Schistosoma mansoni miracidia
}

\author{
Di Liang ${ }^{1}$, Min Zhao', Tianfang Wang ${ }^{1}$, Donald P. McManus ${ }^{2}$ and Scott F. Cummins ${ }^{1 *}$
}

\begin{abstract}
Background: Schistosoma species are responsible for the disease schistosomiasis, a highly prevalent helminthic disease that requires a freshwater snail as intermediate host. The $S$. mansoni free-living miracidium must utilize olfaction to find a suitable snail host, and certain types of rhodopsin G protein-coupled receptors (GPCRs) and ionotropic receptors (IRs) have been identified as olfactory receptors in other animal phyla. The Schistosoma genome project, together with the recent availability of proteomic databases, allowed for studies to explore receptors within S. mansoni, some of which may contribute to host finding.

Results: We have identified 17 rhodopsin-type GPCR sequences in S. mansoni belonging to four subclasses, including ligand-specific GPCRs (i.e. neuropeptide and opsin). RT-PCR demonstrated the expression of nine out of the 17 GPCRs in the free-living miracidia, each of which have been characterized for homology to S. haematobium. Among the nine GPCRs, two are predicted as Gq-opsins. We also describe the characterization of a Schistosomaencoded IR based on similarity with other species IR and conservation of IR-like domains. Schistosoma mansoni IR is expressed in miracidia at 3 and $6 \mathrm{~h}$ post-hatch.

Conclusions: The identification of receptors in S. mansoni miracidia, presented here, contributes not only to further understanding of Schistosoma biology and signal transduction but also provides a basis for approaches that may modify parasite behaviour.
\end{abstract}

Keywords: Schistosoma mansoni, G protein-coupled receptor, lonotropic receptor, Biomphalaria glabrata

\section{Background}

The phylum Platyhelminthes contains prominent endoparasites, including the tapeworms and flukes such as Schistosoma spp. [1]. Schistosomes are responsible for the disease schistosomiasis, the most prevalent and important of the parasitic platyhelminthic diseases of humans. Schistosomiasis occurs in 76 countries, affecting approximately 207 million individuals [2], and causing 280,000 deaths per year in sub-Saharan Africa [3]. This represents a serious disease burden to socioeconomic development.

During the course of its life-cycle, Schistosoma mansoni undergoes distinct stages of differentiation, whilst inhabiting three separate environments - freshwater, a molluscan intermediate host, and a vertebrate definitive host [4]. There are few reports that exist regarding how schistosome

\footnotetext{
* Correspondence: scummins@usc.edu.au

${ }^{1}$ Faculty of Science, Health and Education, University of the Sunshine Coast, Maroochydore, QLD 4558, Australia

Full list of author information is available at the end of the article
}

species interact with these environments, especially concerning the free-living miracidium stage and its infection of the intermediate snail host. Following hatching from the schistosome egg, a motile miracidium actively seeks its Biomphalaria host, within which it undergoes a series of developmental stages and asexual reproduction [5].

Parasites in general have evolved without many of the mechanisms needed to sustain energy for growth or reproduction without a host, for example, miracidia do not have a gut and rely solely on glycogen stored in their epidermal plate for respiration (aerobic in S. mansoni), and lose their infectivity once stores are depleted [6]. Therefore finding a host within a short amount of time in a potentially large body of water requires the miracidia to have highly adapted sensory mechanisms. Miracidia demonstrate host-seeking behaviors in response to chemosensory cues [1]. However, at 1-3 h post-hatch, miracidia use phototactic and geotactic cues to migrate to snail habitats, and do not respond to host chemosensory cues $[7,8]$. After $1-3$ h post-hatch, host 
attractant biomolecule(s) can be detected, reported to be non-specific small molecular weight biomolecules, and this was supported by experimental assays showing an increase in miracidia turn-back responses [9]. Further, macromolecular glycoconjugates referred to as miracidia-attracting glycoconjugates, have been implicated following an observed induction of changes to miracidial turn-back responses [10, 11]. Overall, these studies indicate that these blood flukes possess the molecular components capable of capturing, and more speculatively, processing environmental signals.

It is believed that elucidation of those molecular components that are critical for miracidial function might eventually lead to novel intervention strategies for schistosomiasis control and elimination [12]. Towards this end, recent studies have slowly unraveled insights into schistosome receptor biology and a broad range of cellular processes, such as interaction, mating and reproduction as well as the host-parasite interplay [13, 14]. G-protein coupled receptors (GPCRs) are the largest family of receptors found in eukaryotes, with more than $40 \%$ of all pharmaceuticals targeting their various subfamilies [15]. Due to the large diversity and expansion of GPCRs between species and their ability to respond to a large selection of ligands, selectivity for GPCR-targeted anthelmintic drugs is very promising $[16,17]$. GPCRs are integral membrane receptors, and respond to a multitude of extracellular ligands to transduce and amplify (or inhibit) intracellular responses involved in metabolism, neuromuscular regulation, endocrine function, vision and olfaction [15]. Key characteristics of GPCRs are 7-transmembrane spanning helical $\alpha$-chains (which constitute a hydrophobic core domain), an external $\mathrm{N}$-terminus and an intracellular C-terminus [18]. Among the known classes of GPCRs, the rhodopsin-type superfamily accounts for approximately $85 \%$ of all GPCRs within many species [19] and have constituted a target of research for pharmaceuticals with many known antagonists [20-22]. Rhodopsin-type receptors are activated by a wide range of stimulants, including light, odorant molecules and neurotransmitters, and play physiological roles in vision and smell.

The availability of whole genome sequencing data has provided a basis for the in silico accumulation and analysis of undiscovered and potentially novel receptors in S. mansoni [23]. This led to the description of $117 \mathrm{GPCR}$ genes belonging to five major families (105 Rhodopsin, 2 Glutamate, 3 Adhesion, 2 Secretin and 5 Frizzled) within the draft S. mansoni genome [17]. In 2011, the S. mansoni draft genome set was systematically upgraded with more than $45 \%$ of predicted genes extensively modified and the total number reduced from 11,807 to 10,852 [24]. Employing comparative genomics, platyhelminth GPCRs have been identified and characterized for $S$. mansoni and S. haematobium [25]. Of those, the opsins are rhodopsin-type GPCRs that were inferred to be involved in photoreception, typically thought of as lightabsorbing proteins that act as light sensors in animals [26-28]. Similar to other GPCRs, opsins have a 7-TM structure but are distinguishable from other GPCRs by a lysine residue in the seventh TM domain that binds to retinal, important for light absorption [29]. Upon light absorption, they can transform photons of light into electrochemical signals via $\mathrm{G}$ protein activation [30].

The first stage of eumetazoan animal chemoreception is controlled by chemosensory neurons present within the sensory epithelium, where they express olfactory receptors devoted to binding environmental odorants and transfer this information intracellularly. The accuracy of odor discrimination depends on the specificity with which odorants interact with appropriate olfactory neuronal receptors, which are often rhodopsin GPCRs [31]. The identification of rhodopsin-type GPCRs has been well studied for their role in odor detection in different animals (including humans, mouse, fruit fly, nematode and sea slug), greatly improving our understanding of the molecular mechanism of olfaction in these species [32-37]. In contrast, there is limited information on how olfaction works at the molecular level in the platyhelminths, although GPCRs have been identified as important parasite receptors with potential functions in the tegumental matrix of S. mansoni $[38,39]$.

In the animal kingdom, besides members of the superfamily of GPCRs, it has been found that ionotropic receptors (IRs) can be expressed on the olfactory sensory neurons to help confer olfactory specificity through responses to chemosensory cues [40, 41]. Unlike GPCRs, the characteristic hallmarks of IRs are their three membrane-spanning segments, a pore-forming domain and a ligand-binding Venus flytrap domain, which seems to interact with olfactory stimuli [42]. In insects, IR92a and IR76b are known to detect small amines and polyamines, respectively [43, 44].

In this study, we have used a combination of bioinformatics tools on the improved genomic database to identify S. mansoni GPCR genes, including opsins and putative neuropeptide GPCRs. Importantly, some are expressed in the free-living miracidium, and are possibly involved in host recognition. We also report the characterization of a schistosome-encoded IR. The identification of these receptors not only provides molecular evidence for a potential host recognition strategy in S. mansoni, but also contributes to the understanding of schistosome receptor biology.

\section{Methods}

\section{Identification of putative GPCRs within the S. mansoni genome}

The S. mansoni protein dataset used in this study was based on the improved genome assembly [24], along 
with expression data provided by the GeneDB (www.genedb.org) and SchistoDB (www.schistodb.net) databases. To these databases, we applied Pfam-based profile searches and identification of TM domains with the goal of identifying receptors belonging to the rhodopsin GPCR family. Specifically, this included two bioinformatic tools to predict TM domains for all proteins, including TMHMM (http://www.cbs.dtu.dk/services/TMHMM-2.0/) and Phobius (http://phobius.sbc.su.se/). As TM domains are convenient markers for GPCRs, we only focused on those sequences with 7-TM domains. Next, we applied a Pfam-based profile search using HMMerSearch (http:// hmmer.org/). Proteins containing putative rhodopsintype GPCR domains were systematically identified by profile hidden Markov model searches using the HMMer package (http://hmmer.org/) and the PFAM model PF00001 (7tm_1). Gene and protein nomenclature was based upon the Schistosoma gene models created from the GeneDB reference (www.genedb.org).

\section{Isolation of S. mansoni miracidia}

Livers were obtained from ARC Swiss mice infected with S. mansoni (Puerto Rican strain), under conditions specified by the Australian Department of Agriculture, Fisheries and Forestry (DAFF). A 2-day protocol was used to obtain relatively clean schistosome eggs and miracidia [45]. In brief, the mixture of eggs and mouse liver tissue were incubated with collagenase $\mathrm{B}$, penicillin and streptomycin at $37{ }^{\circ} \mathrm{C}$ overnight, followed by fractionation using Percoll columns $(8 \mathrm{ml}$ Percoll $+32 \mathrm{ml}$ of $0.25 \mathrm{M}$ sucrose in $50 \mathrm{ml}$ tubes). The egg pellets were washed using PBS containing EDTA and EGTA twice on a second Percoll column $(2.5 \mathrm{ml}$ Percoll $+7.5 \mathrm{ml} 0.25 \mathrm{M}$ sucrose in a $15 \mathrm{ml}$ tube). Purified eggs were transferred into a $200 \mathrm{ml}$ hatching measuring cylinder wrapped completely in light-blocking black tape with the exclusion of the top $4 \mathrm{~cm}$ from the lip, thereby producing a light-gradient. The hatching cylinder was topped with $\mathrm{pH}$ neutral spring water until above the tape-covered area $\sim 1.5 \mathrm{~cm}$ and exposed to bright light at $27^{\circ} \mathrm{C}$. Eggs were incubated for $3 \mathrm{~h}$ post-hatch, and the top $10 \mathrm{ml}$ of miracidia-containing water (MCW) was collected for miracidia isolation; in addition, another collection was performed at $6 \mathrm{~h}$ post-hatch. Hatched miracidia were isolated by centrifugation at $8000 \times g$ for $1 \mathrm{~min}$ at $4{ }^{\circ} \mathrm{C}$, and were then washed twice with water. For light microscope examination, $6 \mathrm{~h}$ post-hatch miracidia were fixed in $4 \%$ paraformaldehyde on a slide, dried and washed in PBS before photographs were taken using an Olympus BX60 with Nomarski optics and a Nikon Digital Sight DS-U1 camera. For RNA isolation, miracidia were collected at 3 and $6 \mathrm{~h}$ posthatch and stored separately in RNAlater.
Reverse-transcription PCR for S. mansoni GPCRs

Total RNA was isolated from S. mansoni miracidia (3 and $6 \mathrm{~h}$ post-hatch) using TRIzol reagent (Invitrogen, Carlsbad, CA, USA) and RNA quantity and quality were assessed using UV spectrophotometry (NanoDrop ND1000). First-strand cDNA was generated using random hexamer primers and the Superscript Preamplification System for First-strand Synthesis (Invitrogen). PCR was performed using primers designed (Table 1) on the CLC Genomics Workbench (v6.0; Finlandsgade, Denmark). Amplification of $\alpha$-tubulin served as an internal control for the amount of RNA from each sample. Samples were heated at $94{ }^{\circ} \mathrm{C}$ for $5 \mathrm{~min}$ and amplified for 30 cycles (95 ${ }^{\circ} \mathrm{C}$ for $30 \mathrm{~s}, 45{ }^{\circ} \mathrm{C}$ for $50 \mathrm{~s}$, and $72{ }^{\circ} \mathrm{C}$ for $1 \mathrm{~min}$ ), followed by a $10 \mathrm{~min}$ extension at $72{ }^{\circ} \mathrm{C}$. Reverse transcriptase negative controls were included to detect contaminating genomic DNA. The amplified DNA fragments were analyzed by $2.0 \%(w / v)$ agarose gel electrophoresis.

\section{Comparative analysis of S. mansoni and S. haematobium GPCRs}

Multiple sequence alignments for non-opsin GPCRs were generated with Molecular Evolutionary Genetics Analysis (MEGA) software version 6.0 [46] with the MUSCLE algorithm [47]. Phylogenetic trees were constructed using the neighbor-joining method with 1000 bootstrap replicates for node support. For opsin GPCRs, a phylogenetic tree was constructed on the MEGA 6.0 platform. ClustalW [48] was used to align the sequences of the predicted proteins and the tree was constructed using the neighbor-joining and maximum-likelihood method, with 1000 bootstrap replicates for node support. Neighbor-joining and maximum-likelihood analysis was performed using no. of differences and Jones-TaylorThornton (JTT) method, respectively. Receptor schematic diagrams were prepared using the HMMTOP server version 2.0 (http://www.enzim.hu/hmmtop/html/ document.html) [49] and LaTEX TEXtopo package [50].

\section{Identification and reverse-transcription PCR (RT-PCR) of S. mansoni IR}

The Drosophila melanogaster IR25a [42] was used for sequence similarity searches using the NCBI tBLASTx search tool, limited to bilateria and the nucleotide dataset, resulting in identification of an EST encoding a potential IR protein within S. mansoni. This protein was loaded into the Pfam database (https://www.ebi. ac.uk/Tools/hmmer/search/phmmer and http://pfam. xfam.org/search), which revealed conserved ligandgated ion channel structure. The presence of recurrent TM domain motifs was searched by TMHMM Server v2.0 (http://www.cbs.dtu.dk/services/TMHMM/). Multiple sequence alignments were performed using the MUSCLE algorithm [47] with the MEGA software 
Table 1 Primers used for RT-PCR of GPCR genes in S. mansoni

\begin{tabular}{|c|c|c|c|}
\hline Gene ID & Forward & Reverse & $\mathrm{bp}$ \\
\hline Smp_170020 & GAGATGACTATAAGCG & CAAGTCTGGTTGTATG & 303 \\
\hline Smp_120620 & CGATGCGATAAAGATG & CTGTTAGTGTTGGATG & 471 \\
\hline Smp_141880 & TGTTAGGATGTTAGTGG & GGTTTTAGGTTGCTTG & 345 \\
\hline Smp_104210 & GGCTTGTGAGCTGTATGG & GATGTATGAAAACCTTCGGG & 321 \\
\hline Smp_178420 & ACATACCTACACCACTTCTTC & TTTGTTTGTGCAATCGC & 218 \\
\hline Smp_180030 & ATCAATACTGGCCATGGG & CATAACGTCCAAAACCAAAG & 230 \\
\hline Smp_041880 & TGATTTCTTGGACGTGG & GCTGGTAATAAAGCTGTTC & 478 \\
\hline Smp_148210 & TTGGTGAAGGTATGTGTG & CAGCATGTGCCATATTAC & 365 \\
\hline Smp_043260 & AAAGGCGAATTGGAAAC & CAATCACATATAGCTTCACC & 387 \\
\hline Smp_149580 & CGTTAATTGTATGCTGTTG & TTGGGTTGGTTGTAATGG & 281 \\
\hline Smp_118040 & GGCCTAAAGAATCATCAC & GTACATCCATAACCATCC & 275 \\
\hline Smp_204230 & CACGTAATTGGTCTGTTG & CACATATAACTGGACCAC & 294 \\
\hline Smp_126730 & AGTGAATTGTCCAGAGAAG & GTAGACCGTATTGAGCAG & 358 \\
\hline Smp_173010 & GCTGTATTTCGTGTTCTC & GTAGGATITGTGGATTGG & 392 \\
\hline Smp_203500 & GTAATTTGTGTTCCGTCC & TGTCGATTATCTCTTGCG & 383 \\
\hline Smp_043320 & СТСССТССАААТССТАТС & ATTATCCAAGCACCTCCA & 371 \\
\hline Smp_145520 & ATGATTGGAGGTGCTTGG & GTGTGTTAGAATGCTTCGG & 303 \\
\hline tubulin & GGCGGTGGTACTGGTTCTGGG & CATTTAGCGCACCATCGAAGC & 324 \\
\hline
\end{tabular}

(version 5.1) [46], and the LaTEX TEXtopo package [50] was used to generate schematics showing amino acid conservation.

Isolation of total RNA from $S$. mansoni miracidia followed by the two-step RT-PCR was performed in a similar manner as describe above for the GPCRs. Primers were designed for $S$. mansoni IR (sense, 5'-AGT AGA ATG CGT GAA TGG-3' and antisense, 5'-GTT GCG GTG GTA GTC TTG-3'). Samples were heated at $94{ }^{\circ} \mathrm{C}$ for $5 \mathrm{~min}$ and amplified for 30 cycles $\left(95^{\circ} \mathrm{C}\right.$ for $50 \mathrm{~s}, 46^{\circ} \mathrm{C}$ for $90 \mathrm{~s}$, and $72{ }^{\circ} \mathrm{C}$ for $60 \mathrm{~s}$ ), followed by a 10 min extension at $72{ }^{\circ} \mathrm{C}$. PCR products were visualized by $2.0 \%$ agarose gel electrophoresis to confirm transcript expression.

\section{Molecular dynamics simulation for S. mansoni IR}

The initial conformations of the receptors were built using SWISS-MODEL by sequence alignment of proteins with known 3D structures (template proteins) [51]. The structure with the highest quality estimation, based onQMEAN score, was chosen and subjected to molecular dynamics simulation (MDS) using AMBER version 14. The structure was imported using the LEAP module of AMBER; the sequence segment(s) that was missrepresented (normally at the $\mathrm{N}$ - or $\mathrm{C}$ - terminus), due to different sequence lengths of the template proteins, was built as a linear structure using LEAP and linked back to the corresponding positions. MDS was fully unrestrained and carried out in the canonical ensemble using the SANDER module. The ff14SB force field [52] was employed. Energy minimisation with 2500 steps was first performed to remove unfavourable contacts. The AMBER structure was then heated to $325 \mathrm{~K}$ over $50 \mathrm{ps}$ to avoid being kinetically trapped in local minima [53], then subjected to unrestrained MD simulations at $325 \mathrm{~K}$ for the purpose of peptide equilibration. The structural information was sampled every 1 ps (i.e. 10,000 structures were calculated for $10 \mathrm{~ns}$ MD simulation). This MD simulation was continued until the root mean square deviation of structures within a reasonable long time range was stable at/less than $3 \sim 4 \AA$. Then a lowest energy structure was determined and considered as the representative of the conformations simulated over this period. Visualization of the systems was effected using VMD software [54].

\section{Results}

Putative GPCRs within the S. mansoni genome

Using the methodology outlined in Fig. 1a, 98 proteins with 7-TM domains were extracted from the S. mansoni genome-derived protein models, based on TMHMM prediction. By comparison, Phobius prediction led to the identification of 62 proteins with 7-TM domains. Pfam profiling did classify 87 proteins (E-value $<0.0004$ ) as rhodopsintype receptors. All TMHMM, Phobius and HMMer search results can be found in the Additional file 1: Table S1.

In total, 17 genes encoding class A GPCR-like proteins (326 to 585 amino acids) were identified (Fig. 1b and Table 2) belonging to four subclasses (amine, peptide, opsin and orphan). All encode proteins considered as full-length, as determined by the presence of 7-TM domains, putative rhodopsin-type GPCR domains, as 


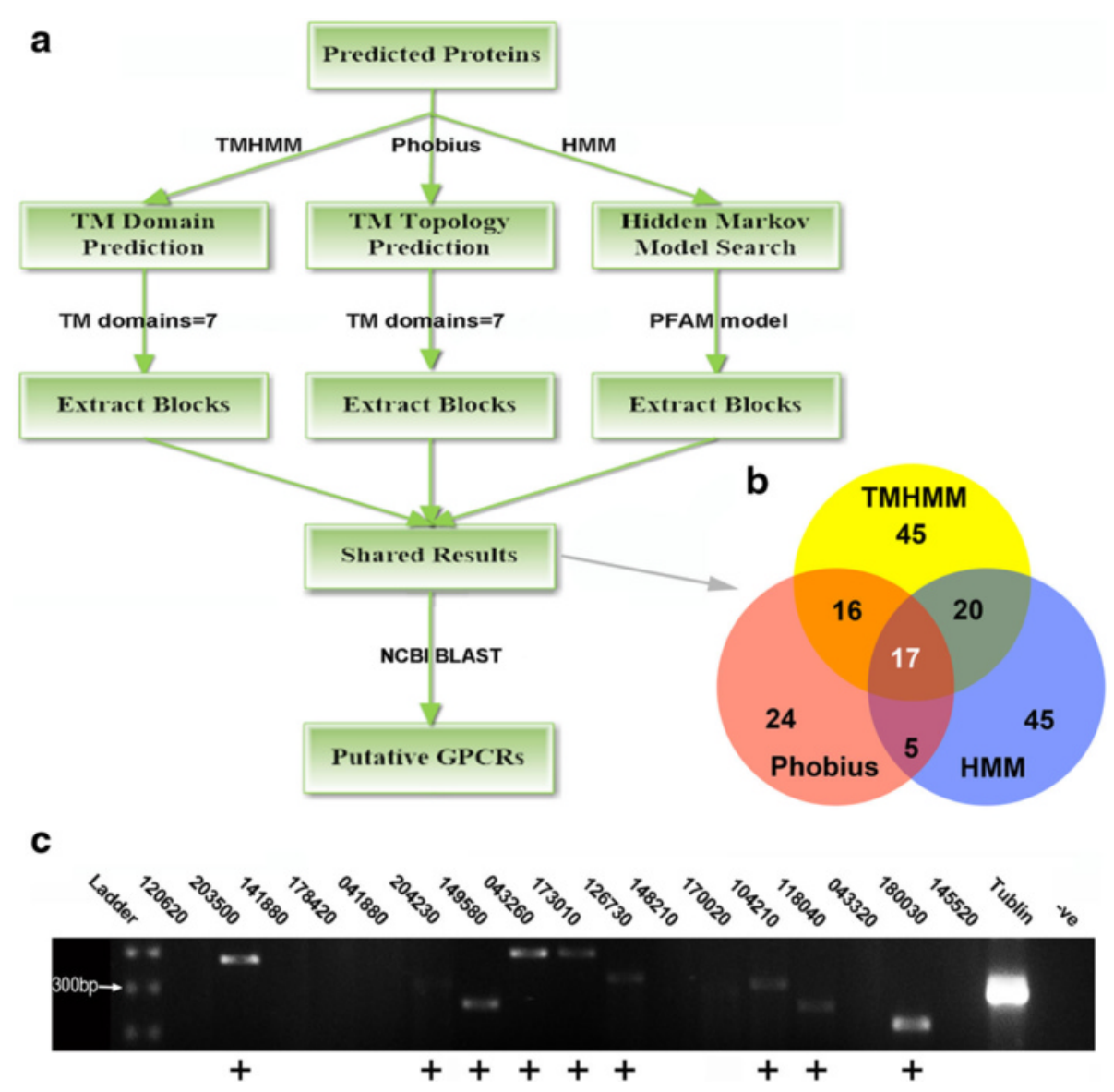

Fig. 1 Candidate GPCRs identified from the S. mansoni genome. a Workflow for mining candidate GPCRs from the $S$. mansoni genome. b Venn diagram showing comparison of three bioinformatics tools (see Methods) listed to define GPCRs. c RT-PCR detection of S. mansoni miracidium GPCR transcripts at $6 \mathrm{~h}$ post-hatch. +, represents presence of amplicon

well as a methionine start and a stop codon. Table 2 also shows the amino acid identity with the identifiable homologs in the closely related S. haematobium [25]. Of the three new orphan GPCRs described in this paper, (i) Smp_203500 shares significant homology (95\% identity) with an allatostatin-A receptor (GenBank XP_012796783.1), (ii) Smp_204230 shares significant homology (87\% identity) with a S. haematobium hypothetical protein (GenBank XP_012798047.1), and (iii) Smp_178420 also shares significant homology (85 \% identity) with a $S$. haematobium hypothetical protein (Genbank XP_012791982.1). RT-PCR of S. mansoni miracidia, using pooled samples obtained at 3 and $6 \mathrm{~h}$ post-hatch, revealed expression of 9 out of the 17 GPCRs (Fig. 1c). Schistosoma mansoni $\alpha$-tubulin was used as a positive control for the cDNA templates.

\section{Comparative analysis of GPCRs present in S. mansoni miracidia with $\mathrm{S}$. haematobium}

According to their corresponding sub-classification, phylogenetic trees were constructed for each subclass using the final set of predicted non-opsin GPCRs grouped with the S. haematobium homologs (Fig. 2), confirming the high phylogenetic similarity. Schematic GPCR representations show specific regions of conservation and divergence between species homologs. Most sequence divergence was noted between the orphan receptors Smp_173010 and Sha_107429, specifically within the region containing the TM6 domain through the C-terminus. The amine-type GPCRs showed most variability within the intracellular loop 3 region. Overall, there was very high conservation observed within the peptide-type GPCRs, although some variability is present within the $\mathrm{N}$-termini region.

Amongst the $S$. mansoni rhodopsin-type GPCR genes, two sequences (Smp_104210 and Smp_180030) were predicted as opsin GPCRs. These encode opsin-like receptors that share the greatest degree of conservation to two $S$. haematobium sequences (Sha_101185 and Sha_101097) (Table 2). We confirmed the clustering of four distinct ancient bilaterian opsin subfamilies (Fig. 3a), namely the Gq-opsins, ciliary opsins, Go-opsins as well as members 
Table 2 NCBI blast match and identity with the GPCRs for S. mansoni

\begin{tabular}{|c|c|c|c|c|c|}
\hline Protein name & $\begin{array}{l}\text { Aligned to } S \text {. } \\
\text { haematobium }\end{array}$ & Overall identity (\%) & GPCR subclass [17] & NCBI BLAST best hit (Reference/Name/Species) & Identity (\%) \\
\hline Smp_043320 & Sha_102744 & 75 & amine & emb|CCD79388.1| Tyramine-like receptor [S. mansoni] & 100 \\
\hline Smp_126730 & Sha_Exo_8 & 94 & amine & emb|CCD75608.1| 5-HT-like receptor [S. mansoni] & 100 \\
\hline Smp_145520 & Sha_104648 & 53 & amine & emb| CCD79384.1| Putative adrenoreceptor [S. mansoni] & 100 \\
\hline Smp_148210 & Sha_101211 & 33 & amine & emb|CCD77402.1| 5-HT-like receptor [S. mansoni] & 100 \\
\hline Smp_043260 & Sha_Exo_1 & 87 & amine & emb|CCD79382.1| Putative GPCR receptor [S. mansoni] & 100 \\
\hline Smp_141880 & Sha_101834 & 76 & peptide & emb|CCD81490.1| Neuropeptide-like receptor [S. mansoni] & 100 \\
\hline Smp_118040 & Sha_108334 & 86 & peptide & emb|CCD75717.1| Neuropeptide-like receptor [S. mansoni] & 100 \\
\hline Smp_041880 & Sha_104441 & 88 & peptide & emb|CCD79664.1| Peptide-like receptor [S. mansoni] & 100 \\
\hline Smp_149580 & Sha_101834 & 80 & peptide & emb|CCD59781.1| FMRFamide-like receptor [S. mansoni] & 100 \\
\hline Smp_170020 & Sha_104691 & 86 & peptide & emb|CCD82074.1| Neuropeptide-like receptor [S. mansoni] & 100 \\
\hline Smp_120620 & Sha_102107 & 19 & peptide & emb|CCD59108.1| Amine GPCR-like receptor [S. mansoni] & 100 \\
\hline Smp_104210 & Sha_101185 & 80 & opsin & gb|AAF73286.1| Opsin-like receptor [S. mansoni] & 100 \\
\hline Smp_180030 & Sha_101097 & 85 & opsin & emb|CCD82678.1| Opsin-like receptor [S. mansoni] & 100 \\
\hline Smp_173010 & Sha_107429 & 66 & orphan & emb|CCD76373.1| Myosuppressin-like receptor [S. mansoni] & 100 \\
\hline Smp_204230 & Sha_105544 & 87 & orphan & ref|XP_012798047.1| Hypothetical protein [S. haematobium] & 87 \\
\hline Smp_178420 & Sha_108078 & 85 & orphan & ref|XP_012791982.1| Hypothetical protein [S. haematobium] & 85 \\
\hline Smp_203500 & Sha_105605 & 95 & orphan & ref|XP012796783.1| Allatostatin receptor [S. haematobium] & 95 \\
\hline
\end{tabular}

of the retinal-photoisomerase subfamily, which includes retinal GPCR (RGR) and retinochrome. As might be expected, Smp_104210 and Smp_180030 grouped with Sha_101185 and Sha_101097 in a pairwise, orthologous manner within the Gq-opsin group. Partial sequence alignment of members of the Gq-opsin subfamilies, specifically within the cytosolic region of the TM 7 domain and C-terminal tail, demonstrates two highly conserved peptide amino acids [Histidine, Proline $(\mathrm{H}, \mathrm{P})$ ] in the carboxy terminal intra-cellular loop domain that are highly indicative of Gq-opsin families (Fig. 3b, c). This distinctive characteristic is conserved in both $S$. mansoni Smp_104210 and S. haematobium Sha_101185. No such motif was detected for the other opsin-like GPCR (Smp_180030 and Sha_101097).

\section{Identification of IRs within S. mansoni}

A single $S$. mansoni $I R$ was identified from the genome that encodes a conventional ligand-gated ion channel domain protein (513 aa; $58.7 \mathrm{kDa})$. This receptor, $S$. mansoni IR, displays remnants of classical IR motifs at corresponding positions and predicted domains that are critical structural regions responsible for detecting odor ligands and contributing to ligand specificity, including an extracellular two-lobed ligand-binding domain and four features common to all conventional IRs, namely: (i) IR-related motifs with TM stretches, (ii) possession of Pfam domains PF10613 and PF00060, which are specific for the ligand-gated ion channel receptors, (iii) highly-conserved structural features specifically shared amongst the IR family, and (iv) a region surrounding the ligand-binding domain. All are present within the $S$. mansoni IR receptor showing considerable conservation with the IRs of other species, including the well-studied D. melanogaster.

A representation of the $S$. mansoni IR compared to six Protostomia species, including Panulirus argus, Helicoverpa assulta, Microplitis mediator, Dendroctonus ponderosae, Schistocerca gregaria and Drosophila melanogaster was used to unify protein structure predictions across species (Fig. 4a). Sequences used for this analysis are provided in Additional file 2: Table S2. All IRs display classical IR motifs at corresponding positions and critical structural regions responsible for binding ligands and contributing to ligand specificity. Figure $4 \mathrm{~b}, \mathrm{c}$ demonstrates the proposed structure model of the Venus flytrap domain of the schistosome IR, and with the putative ligand binding sites. RT-PCR results demonstrate $S$. mansoni IR expression within the free-living miracidia at both 3 and 6 h post-hatch (Fig. 4d).

\section{Discussion}

Schistosome miracidia must find an appropriate host within a very limited time-span, thus it would seem advantageous for them to have evolved finely-tuned molecular strategies allowing for host detection, thereby increasing the likelihood of successful snail infection. GPCRs and IRs are fundamental to chemoreception in many animal species [55-57], and we speculate this may also be similar for the Schistosoma miracidia. In this 


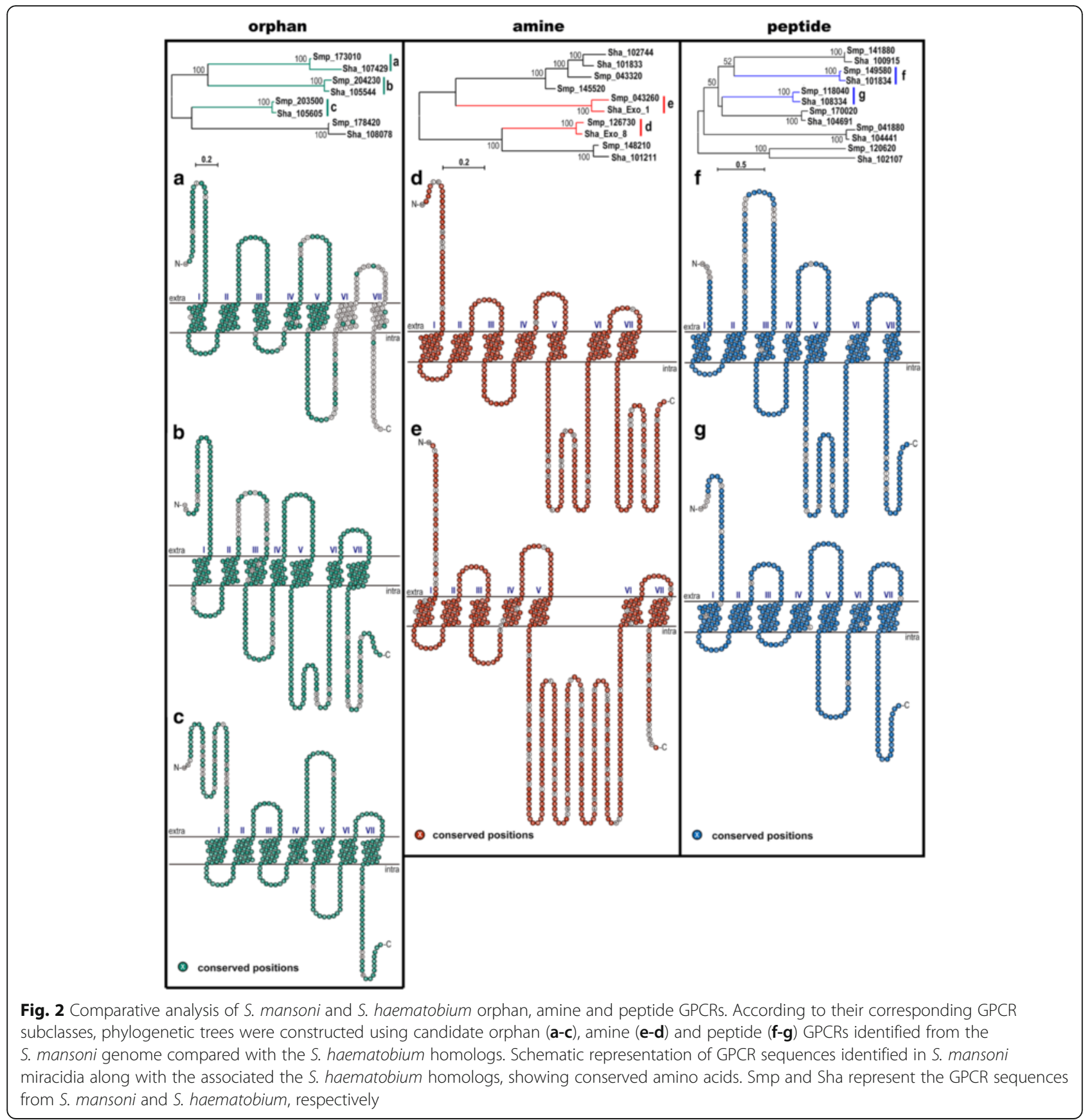

study, we have analyzed the $S$. mansoni genome to identify putative receptors, and specifically those present within the miracidium, some of which may be important for snail host-finding.

We identified 17 rhodopsin-type GPCRs that belong to amine, peptide, opsin and orphan groups. Among these, the amine group consisted of biogenic amine receptors such as serotonin, dopamine and histamine, that have a prominent role in the flatworm nervous system [58, 59]. Of these, experimental validation has been established for the histamine receptor (Smp_043260) [60]. Regarding the other GPCRs identified in this study, three (Smp_203500,Smp_204230 and Smp_178420) have not been described previously, while Smp_173010 was reported by Campos et al. [25] as novel platyhelminthspecific rhodopsin-like orphan family (PROF). This variation can be explained by the different workflow for curation of the final GPCR list, whereby receptors were only taken if they satisfied requirements within all of TMHMM, HMM and Phobius tools. Smp_203500 and 

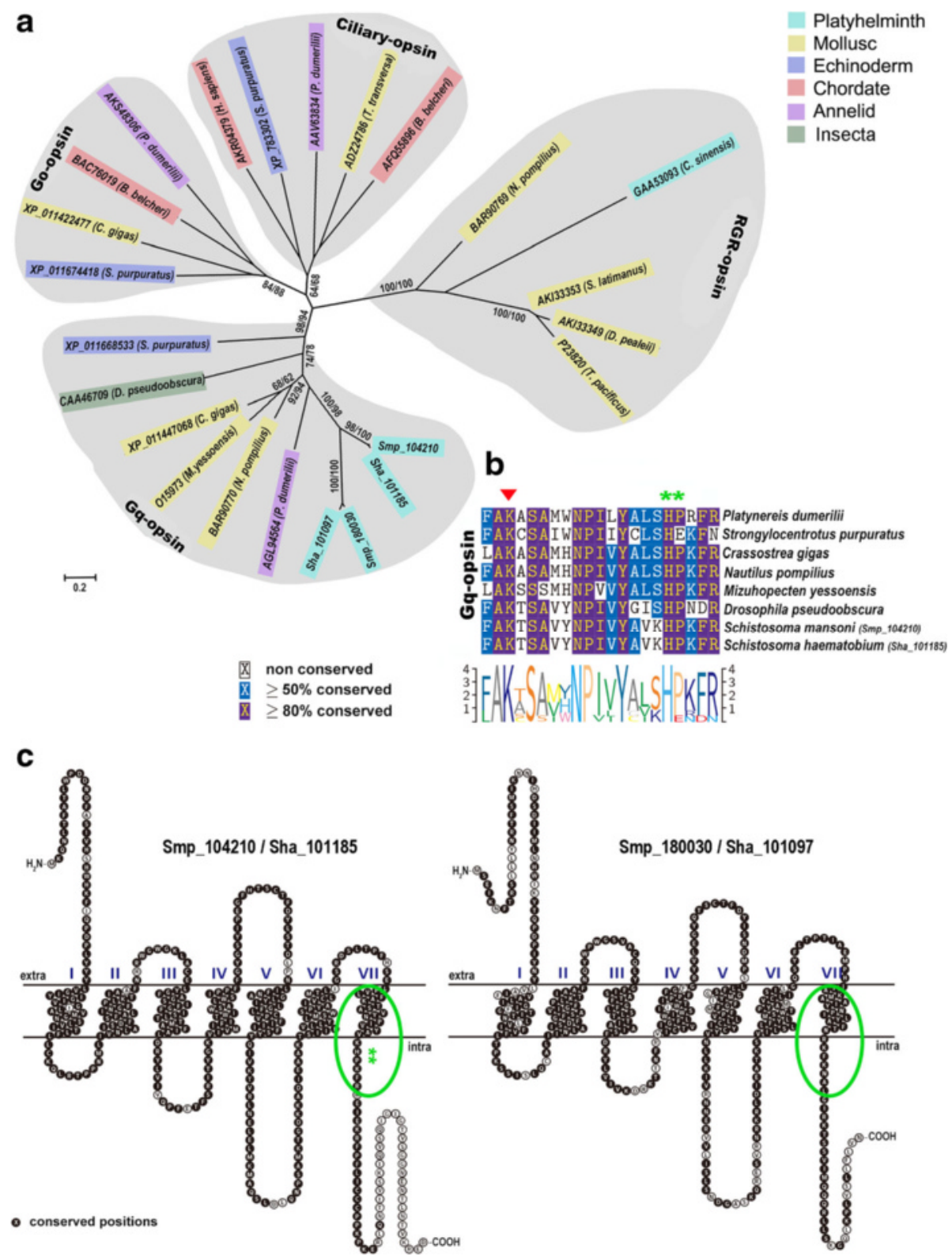

Fig. 3 Phylogenetic analysis and characterization of opsins. a Phylogeny showing that representative bilaterian opsin members cluster into four supported subfamilies. Smp, S. mansoni and Sha, S. haematobium. Branch support values are indicated next to branching points. Maximum-likelihood bootstrap support values (significant support threshold value $>60 \%$ ) are indicated as the first set of numbers at the nodes. Neighbor-joining bootstrap support values are indicated as the second set of numbers at the nodes, with bootstrap value expressed values above $60 \%$ shown. Scale-bar indicates the relative amount of amino acid changes. $\mathbf{b}$ Partial amino acid alignment of members of the Gq-opsin subfamilies. Asterisks demarcate the fingerprint indicative of Gq-opsin families. An arrowhead demarcates the position of the lysine residue critical for Schiff base formation. c Schematic representation of S. mansoni opsin compared with the S. haematobium homologs. Circle shows region from panel $\mathbf{b}$ and asterisks demarcate presence of the Gq-opsin fingerprint

PROF receptors Smp_173010 do have some similarity to characterized receptor types, allatostatin and myosuppres$\sin$, respectively; however this has not been experimentally validated. Platyhelminthes appear to lack a conventional endocrine system [38], and are therefore heavily reliant on neural signalling via neuropeptides that control vital dynamic physiological and neural functions, such as growth, reproduction, host-seeking olfaction, locomotion, immune evasion and sexual dimorphism [61]. The importance of their peptidergic neural and associated receptor systems holds a promising area of research for new anthelmintic drug targets $[16,62,63]$.

RT-PCR demonstrated the expression of nine out of the 17 GPCRs in the free-living miracidia, suggesting 

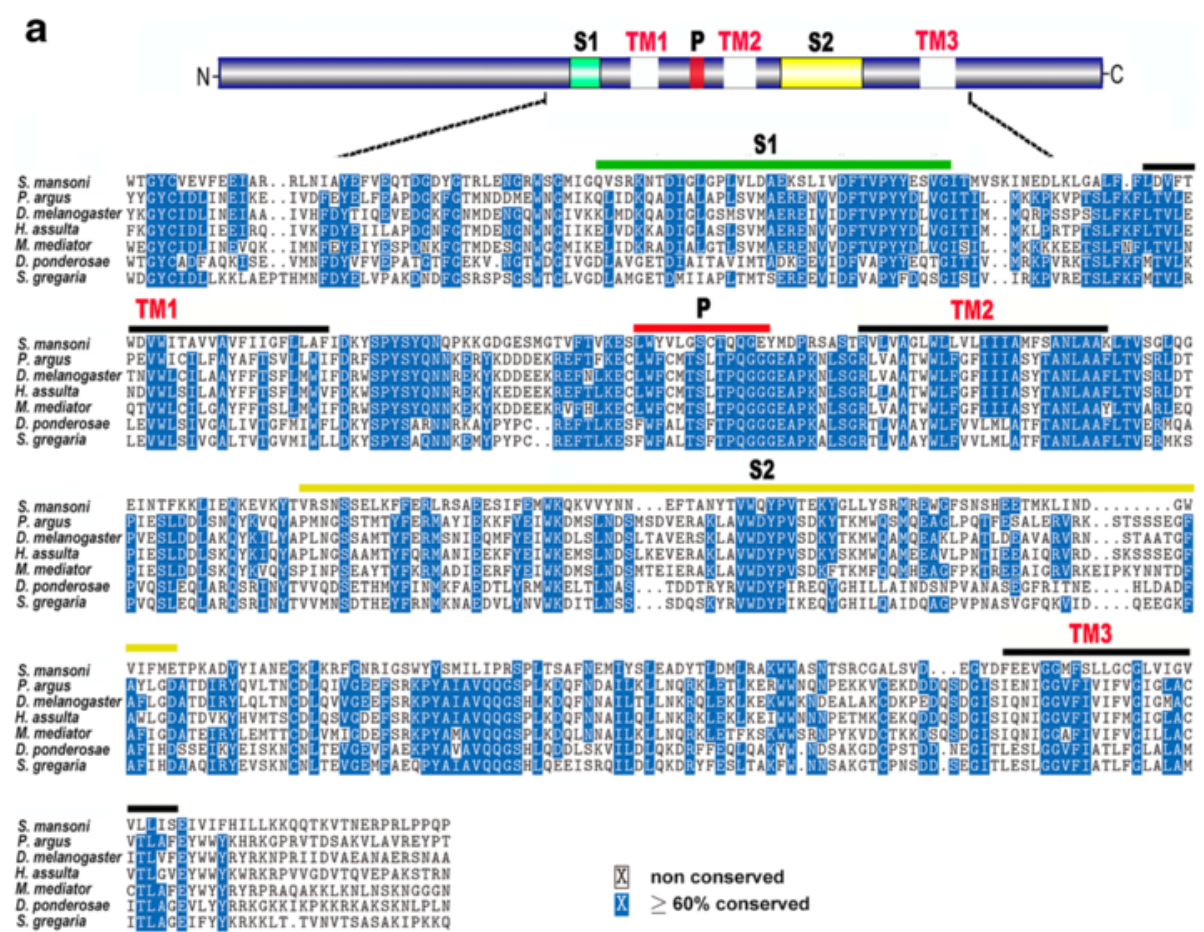

X non conserved

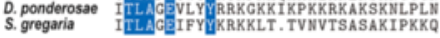

$\geq 60 \%$ conserved

b

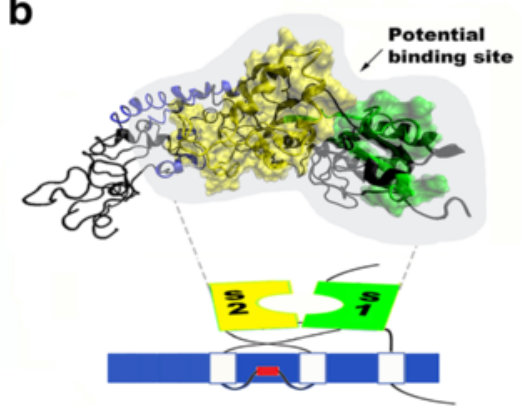

d

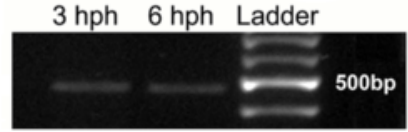

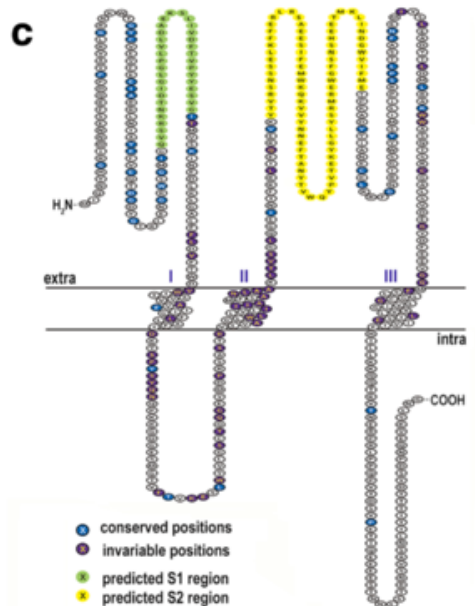

Fig. 4 Characterisation of S. mansoni IR. a Sequence analysis of Schistosoma IR and in comparison with IR from other species. Top: The predicted protein domain organization of typical IR is shown in histogram. Below: Alignment of Schistosoma IR with IR proteins of representative selections from other species. Domains: TM, transmembrane domain; S, ligand-binding domain; P, pore. b Structure of S. mansoni IR as predicted by MDS model with space filling of predicted binding sites. Yellow shows the predicted ligand binding S1 region, green shows the predicted ligand binding S2 region, and blue demarcates the predicted TM regions. The protein domain structure of conventional IRs in cartoon form is shown below [40, 41]. c Schematic representation of all IRs shown in a, demonstrating conserved and invariable amino acids, as well as predicted S1 and S2 regions. d RT-PCR showing expression of $\mathbb{I R}$ in S. mansoni miracidia at $3 \mathrm{~h}$ and $6 \mathrm{~h}$ post-hatch (hph)

that these receptors are possibly either involved in miracidia host-finding and recognition or required for miracidia metabolism, including the histamine receptor. Representative comparative schematic models demonstrated the divergence in amino acid sequence of these GPCRs and homologs in S. haematobium, suggesting the potential biological differences between these two schistosomes. Notably, PROF receptor Smp_173010 shows relatively large variation in protein sequence within the C-terminal region, thus we speculate that there may be binding of species-specific ligands through this receptor.

The opsin-like GPCR Smp_104210 has been reported as being differentially regulated during the parasite's lifecycle and, in the cercaria, it localizes to organelles found directly below the parasite's epidermis, associated with 
organelles within the vicinity of the most anterior osmoregulatory flame cells [64]. Based on this morphological description, it likely acts as a photoreceptor responsible for the direct photokinetic behavior of cercaria in response to light [64]. Further, annotation of opsin-like GPCR Smp_180030 has been determined as a result of the analysis of RNA-seq expression profiles [24], yet no role in schistosome photoreception processes has been reported.

As indicated by our phylogenetic analysis, Smp_104210 and Smp_180030 have well-supported clustering with other Gq-opsin genes involved in photosensation that strongly implies that these receptors are Gq-opsins. The detection of both receptors (Smp_104210 and Smp_180030) in light-responsive miracidia, as well as their clustering with other $\mathrm{Gq}$ opsins, suggests that they may serve an integral role in host-finding by participating in Schistosoma photokinesis [8, 64-66].

We identified a single $S$. mansoni IR. The IR repertoire throughout protostomes shows substantial variation in size, ranging from three in C. elegans to 85 in the crustacean Daphnia pulex [67, 68]. The $S$. mansoni IR exhibits the typical venus flytrap structure (see Fig. 4b) and shares sequence similarity to characterised IRs of other species. Another type of venus flytrap receptor has been studied in $S$. mansomi, known as the venus kinase receptor [69]. There are two isoforms of this receptor, one that binds L-arginine (SmVKR1) and another that binds calcium ions (SmVKR2), which are thought to be important for development and reproduction. Like the IR identified in our study, the ligand for the $S$. mansoni venus kinase receptor is unknown. IRs in the insects are known to bind polyamines [43], yet may also act as thermosensors [70]. We speculate that the $S$. mansoni IR could play an important role as a chemosensory and/or thermosensory receptor in different life-cycle stages, supported by its observed expression profile in cercariae, schistosomula and adults (GeneDB, version 4.0).

\section{Conclusions}

The characterization of GPCRs and IRs in S. mansoni is likely to inform us about their pharmacological profiles and features towards manipulating chemosensorydriven behaviors. Given that S. mansoni IR and at least some of the GPCRs are expressed in the miracidium, we hypothesize they may be dedicated to detect specific odor cues, including responses to odors emitted from Biomphalaria. As these odors are likely instrumental for parasite transmission, GPCRs and IRs may represent proteins against which novel prophylactic therapies can be developed.

\section{Additional files}

Additional file 1: Table S1. TMHMM, Phobius and HMMer search results during the identification of putative GPCRs within the S. mansoni genome. (XLSX 98 kb)

Additional file 2: Table S2. Sequences used for phylogenetic analysis in Fig. 4a. (XLSX 19 kb)

\section{Abbreviations}

GPCRs: G protein-coupled receptors; HMM: Hidden Markov Model; IRs: Ionotropic receptors; MDS: Molecular dynamics simulation; MEGA: Molecular evolutionary genetics analysis; PROF: Platyhelminth-specific rhodopsin-like orphan family; RGR: Retinal GPCR; TM: Transmembrane

\section{Acknowledgments}

We acknowledge the help of Ms Mary Duke of QIMR Berghofer with the egg hatching and the miracidia processing and harvesting. This research was undertaken with the assistance of resources from the National Computational Infrastructure (NCl), which is supported by the Australian Government.

\section{Funding}

This work was supported by the Australian Research Council (Future Fellowship, FT110100990 to SFC). We thank the University of the Sunshine Coast who provided an internal grant to help support this work (TW). The funders had no role in study design, data collection and analysis, decision to publish, or preparation of the manuscript.

\section{Availability of data and material}

The original S. mansoni protein dataset is available from the GeneDB (www.genedb.org) and SchistoDB (www.schistodb.net) databases.

\section{Authors' contributions}

Conceived and designed the study and supervised the project: SFC and MZ. Undertook the study and data analysis: $\mathrm{DL}, \mathrm{MZ}$ and TW. Contributed to analysis using various tools: DL, MZ and TW. Wrote the paper: DL, DPM and SFC. All authors read and approved the final version of the manuscript.

\section{Competing interests}

The authors declare that they have no competing interests.

\section{Consent for publication}

Not applicable.

\section{Ethics approval and consent to participate}

The conduct and procedures involving animal experimentation were approved by the Animal Ethics Committee of the QIMR Berghofer Medical Research Institute (project number P242). This study was performed in accordance with the recommendations in the Guide for the Care and Use of Laboratory Animals of the National Institutes of Health.

\section{Author details}

${ }^{1}$ Faculty of Science, Health and Education, University of the Sunshine Coast, Maroochydore, QLD 4558, Australia. ${ }^{2}$ Molecular Parasitology Laboratory, QIMR Berghofer Medical Research Institute, Brisbane Q4006, Australia.

Received: 19 July 2016 Accepted: 5 October 2016

Published online: 26 October 2016

\section{References}

1. Chaisson KE, Hallem EA. Chemosensory behaviors of parasites. Trends Parasitol. 2012;28(10):427-36.

2. Steinmann $P$, Keiser J, Bos R, Tanner M, Utzinger J. Schistosomiasis and water resources development: systematic review, meta-analysis, and estimates of people at risk. Lancet Infect Dis. 2006;6(7):411-25.

3. van der Werf MJ, de Vlas SJ, Brooker S, Looman CW, Nagelkerke NJ, Habbema JD, et al. Quantification of clinical morbidity associated with schistosome infection in sub-Saharan Africa. Acta Trop. 2003;86(2-3):125-39.

4. Wajdi N. Penetration by the miracidia of S. mansoni into the snail host. Helminthol. 1966;40(1):235-44. 
5. Cheng TC, Bier JW. Studies on molluscan schistosomiasis: an analysis of the development of the cercaria of Schistosoma mansoni. Parasitology. 1972; 64(1):129-41.

6. Galaktionov KV, Dobrovolskij A. The biology and evolution of trematodes: An essay on the biology, morphology, life cycles, transmissions, and evolution of digenetic trematodes. Dordrecht: Kluwer Academic Publishers; 2003.

7. Sukhdeo MV, Sukhdeo S. Trematode behaviours and the perceptual worlds of parasites. Can J Zool. 2004;82(2):292-315.

8. Mason PR, Fripp PJ. The reactions of Schistosoma mansoni miracidia to light. J Parasitol. 1977;63(2):240-4.

9. Maclnnis AJ. Responses of Schistosoma mansoni miracidia to chemical attractants. J Parasitol. 1965;51(5):731-46.

10. Kalbe M, Haberl B, Haas W. Miracidial host-finding in Fasciola hepatica and Trichobilharzia ocellata is stimulated by species-specific glycoconjugates released from the host snails. Parasitol Res. 1997:83(8):806-12.

11. Haberl B, Korner M, Spengler Y, Hertel J, Kalbe M, Haas W. Host-finding in Echinostoma caproni: miracidia and cercariae use different signals to identify the same snail species. Parasitology. 2000;120(Pt 5):479-86.

12. Hagan $\mathrm{P}$, Doenhoff MJ, Wilson RA, Al-Sherbiny M, Bergquist $R$. Schistosomiasis vaccines: a response to a devils' advocate's view. Parasitol Today. 2000;16(8):322-3.

13. Boissier J, Mone H. Male-female larval interactions in Schistosoma mansoniinfected Biomphalaria glabrata. Int J Parasitol. 2001;31(4):352-8.

14. Jones MK, Gobert GN, Zhang L, Sunderland P, McManus DP. The cytoskeleton and motor proteins of human schistosomes and their roles in surface maintenance and host-parasite interactions. Bioessays. 2004;26(7):752-65.

15. Chung KY. Structural aspects of GPCR-G protein coupling. Toxicol Res. 2013;29(3):149-55.

16. McVeigh P, Atkinson L, Marks NJ, Mousley A, Dalzell JJ, Sluder A, et al. Parasite neuropeptide biology: Seeding rational drug target selection? Int J Parasitol Drugs Drug Resist. 2012;2:76-91.

17. Zamanian M, Kimber MJ, McVeigh P, Carlson SA, Maule AG, Day TA. The repertoire of $\mathrm{G}$ protein-coupled receptors in the human parasite Schistosoma mansoni and the model organism Schmidtea mediterranea. BMC Genomics. 2011;12:596.

18. Moreira IS. Structural features of the G-protein/GPCR interactions. Biochim Biophys Acta. 2014;1840(1):16-33.

19. Zhang Z, Wu J, Yu J, Xiao J. A brief review on the evolution of GPCR: conservation and diversification. Open J Genet. 2013;2(04):11.

20. Wise A, Gearing K, Rees S. Target validation of G-protein coupled receptors. Drug Discov Today. 2002;7(4):235-46.

21. Lagerstrom MC, Schioth HB. Structural diversity of G protein-coupled receptors and significance for drug discovery. Nat Rev Drug Discov. 2008;7(4):339-57.

22. Müller CE, Schiedel AC, Baqi Y. Allosteric modulators of rhodopsin-like G protein-coupled receptors: Opportunities in drug development. Pharmacol Ther. 2012;135(3):292-315.

23. Berriman M, Haas BJ, LoVerde PT, Wilson RA, Dillon GP, Cerqueira GC, et al. The genome of the blood fluke Schistosoma mansoni. Nature. 2009; 460(7253):352-8.

24. Protasio AV, Tsai IJ, Babbage A, Nichol S, Hunt M, Aslett MA, et al. A systematically improved high quality genome and transcriptome of the human blood fluke Schistosoma mansoni. PLoS Negl Trop Dis. 2012;6(1):e1455.

25. Campos TD, Young ND, Korhonen PK, Hall RS, Mangiola S, Lonie A, et al. Identification of $\mathrm{G}$ protein-coupled receptors in Schistosoma haematobium and S. mansoni by comparative genomics. Parasit Vectors. 2014;7:242.

26. Shichida $Y$, Imai $H$. Visual pigment: G-protein-coupled receptor for light signals. Cell Mol Life Sci. 1998;54(12):1299-315.

27. Gartner W, Towner P. Invertebrate visual pigments. Photochem Photobiol. 1995;62(1):1-16

28. Sakmar TP, Menon ST, Marin EP, Awad ES. Rhodopsin: insights from recent structural studies. Annu Rev Biophys Biomol Struct. 2002;31:443-84.

29. Terakita A. The opsins. Genome Biol. 2005;6(3):213.

30. Shichida Y, Matsuyama T. Evolution of opsins and phototransduction. Philos Trans R Soc Lond B Biol Sci. 2009;364(1531):2881-95.

31. Breer $\mathrm{H}$. Olfactory receptors: molecular basis for recognition and discrimination of odors. Anal Bioanal Chem. 2003:377(3):427-33.

32. Buck $L$, Axel R. A novel multigene family may encode odorant receptors: a molecular basis for odor recognition. Cell. 1991;65(1):175-87.

33. Troemel ER, Chou JH, Dwyer ND, Colbert HA, Bargmann Cl. Divergent seven transmembrane receptors are candidate chemosensory receptors in $C$. elegans. Cell. 1995;83(2):207-18.
34. Clyne PJ, Warr CG, Freeman MR, Lessing D, Kim J, Carlson JR. A novel family of divergent seven-transmembrane proteins: candidate odorant receptors in Drosophila. Neuron. 1999;22(2):327-38.

35. Vassilatis DK, Hohmann JG, Zeng H, Li F, Ranchalis JE, Mortrud MT, et al. The $G$ protein-coupled receptor repertoires of human and mouse. Proc Natl Acad Sci U S A. 2003:100(8):4903-8.

36. Cummins SF, Erpenbeck D, Zou Z, Claudianos C, Moroz LL, Nagle GT, et al. Candidate chemoreceptor subfamilies differentially expressed in the chemosensory organs of the mollusc Aplysia. BMC Biol. 2009;7:28.

37. Srivastava M, Simakov O, Chapman J, Fahey B, Gauthier ME, Mitros T, et al. The Amphimedon queenslandica genome and the evolution of animal complexity. Nature. 2010;466(7307):720-6.

38. El-Shehabi F, Vermeire JJ, Yoshino TP, Ribeiro P. Developmental expression analysis and immunolocalization of a biogenic amine receptor in Schistosoma mansoni. Exp Parasitol. 2009;122(1):17-27.

39. El-Shehabi F, Taman A, Moali LS, El-Sakkary N, Ribeiro P. A novel G proteincoupled receptor of Schistosoma mansoni (SmGPR-3) is activated by dopamine and is widely expressed in the nervous system. PLoS Negl Trop Dis. 2012;6(2):e1523.

40. Croset V, Rytz R, Cummins SF, Budd A, Brawand D, Kaessmann H, et al. Ancient protostome origin of chemosensory ionotropic glutamate receptors and the evolution of insect taste and olfaction. PLoS Genet. 2010;6(8):e1001064

41. Liu Y, Gu S, Zhang Y, Guo Y, Wang G. Candidate olfaction genes identified within the Helicoverpa armigera antennal transcriptome. PLoS One. 2012; 7(10):e48260.

42. Benton R, Vannice KS, Gomez-Diaz C, Vosshall LB. Variant ionotropic glutamate receptors as chemosensory receptors in Drosophila. Cell. 2009; 136(1):149-62.

43. Hussain A, Zhang M, Ucpunar HK, Svensson T, Quillery E, Gompel N, et al. Ionotropic chemosensory receptors mediate the taste and smell of polyamines. PLoS Biol. 2016;14(5):e1002454.

44. Min S, Ai M, Shin SA, Suh GS. Dedicated olfactory neurons mediating attraction behavior to ammonia and amines in Drosophila. Proc Natl Acad Sci U S A. 2013;110(14):E1321-9.

45. Wang $T$, Zhao M, Rotgans BA, Strong A, Liang D, Ni G, et al. Proteomic analysis of the Schistosoma mansoni miracidium. PLoS One. 2016;11(1): e0147247.

46. Kumar S, Stecher G, Peterson D, Tamura K. MEGA-CC: computing core of molecular evolutionary genetics analysis program for automated and iterative data analysis. Bioinformatics. 2012;28(20):2685-6.

47. Edgar RC. MUSCLE: multiple sequence alignment with high accuracy and high throughput. Nucleic Acids Res. 2004;32(5):1792-7.

48. Thompson JD, Gibson T, Higgins DG. Multiple sequence alignment using ClustalW and ClustalX. Curr Protoc Bioinformatics. 2002:2.3.1-2.3.22. doi: 10 1002/0471250953.bi0203s00

49. Tusnady GE, Simon I. The HMMTOP transmembrane topology prediction server. Bioinformatics. 2001;17(9):849-50

50. Beitz E. T(E)Xtopo: shaded membrane protein topology plots in LAT(E)X2epsilon. Bioinformatics. 2000;16(11):1050-1.

51. Biasini M, Bienert S, Waterhouse A, Arnold K, Studer G, Schmidt T, et al. SWISS-MODEL: modelling protein tertiary and quaternary structure using evolutionary information. Nucleic Acids Res. 2014;42:W252-8.

52. Duan Y, Wu C, Chowdhury S, Lee MC, Xiong G, Zhang W, et al. A pointcharge force field for molecular mechanics simulations of proteins based on condensed-phase quantum mechanical calculations. J Comput Chem. 2003; 24(16):1999-2012.

53. Maisuradze GG, Liwo A, Oldziej S, Scheraga HA. Evidence, from simulations, of a single state with residual native structure at the thermal denaturation midpoint of a small globular protein. J Am Chem Soc. 2010;132(27):9444-52.

54. Humphrey W, Dalke A, Schulten K. VMD: visual molecular dynamics. J Mol Graph. 1996;14(1):33-8. 27-38.

55. Touhara K, Vosshall LB. Sensing odorants and pheromones with chemosensory receptors. Annu Rev Physiol. 2009;71:307-32.

56. Ebrahimi FA, Chess A. Olfactory G proteins: simple and complex signal transduction. Curr Biol. 1998;8(12):R431-3.

57. Wicher D. Functional and evolutionary aspects of chemoreceptors. Front Cell Neurosci. 2012;6:48.

58. Ribeiro P, El-Shehabi F, Patocka N. Classical transmitters and their receptors in flatworms. Parasitology. 2005;131(Suppl):S19-40. 
59. Ribeiro P, Geary TG. Neuronal signaling in schistosomes: current status and prospects for postgenomics. Can J Zool. 2009;88(1):1-22.

60. Hamdan FF, Abramovitz M, Mousa A, Xie J, Durocher Y, Ribeiro P. A novel Schistosoma mansoni $\mathrm{G}$ protein-coupled receptor is responsive to histamine. Mol Biochem Parasitol. 2002;119(1):75-86.

61. Collins JJ, Hou X, Romanova EV, Lambrus BG, Miller CM, Saberi A, et al. Genome-wide analyses reveal a role for peptide hormones in planarian germline development. PLoS Biol. 2010;8(10):e1000509.

62. McVeigh P, Mair GR, Atkinson L, Ladurner P, Zamanian M, Novozhilova E, et al. Discovery of multiple neuropeptide families in the phylum Platyhelminthes. Int J Parasitol. 2009;39(11):1243-52.

63. Ribeiro P, Patocka N. Neurotransmitter transporters in schistosomes: structure, function and prospects for drug discovery. Parasitol Int. 2013;62(6):629-38.

64. Hoffmann KF, Davis EM, Fischer ER, Wynn TA. The guanine protein coupled receptor rhodopsin is developmentally regulated in the free-living stages of Schistosoma mansoni. Mol Biochem Parasitol. 2001;112(1):113-23.

65. Short RB, Gagne HT. Fine structure of possible photoreceptor in cercariae of Schistosoma mansoni. J Parasitol. 1975;61(1):69-74.

66. Saladin KS. Schistosoma mansoni: cercarial responses to irradiance changes. J Parasitol. 1982;68(1):120-4.

67. Corey EA, Bobkov Y, Ukhanov K, Ache BW. Ionotropic crustacean olfactory receptors. PLoS One. 2013;8(4):e60551.

68. Groh-Lunow KC, Getahun MN, Grosse-Wilde E, Hansson BS. Expression of ionotropic receptors in terrestrial hermit crab's olfactory sensory neurons. Front Cell Neurosci. 2014;8:448.

69. Dissous C, Morel M, Vanderstraete M. Venus kinase receptors: prospects in signaling and biological functions of these invertebrate kinases. Front Endocrinol (Lausanne). 2014:5:72.

70. Ni L, Klein M, Svec KV, Budelli G, Chang EC, Ferrer AJ, et al. The ionotropic receptors IR21a and IR25a mediate cool sensing in Drosophila. Elife. 2016:5 doi: 10.7554/eLife.13254

\section{Submit your next manuscript to BioMed Central} and we will help you at every step:

- We accept pre-submission inquiries

- Our selector tool helps you to find the most relevant journal

- We provide round the clock customer support

- Convenient online submission

- Thorough peer review

- Inclusion in PubMed and all major indexing services

- Maximum visibility for your research

Submit your manuscript at www.biomedcentral.com/submit

) Biomed Central 